\title{
An open-label, long-term study examining the safety and tolerability of pregabalin in Japanese patients with central neuropathic pain
}

This article was published in the following Dove Press journal: Journal of Pain Research

28 July 2014

Number of times this article has been viewed

\section{Kenji Onouchi' \\ Hiroaki Koga ${ }^{2}$ \\ Kazumasa Yokoyama ${ }^{3}$ \\ Tamotsu Yoshiyama ${ }^{4}$}

'Aida Memorial Rehabilitation Hospital, Moriya, Japan; ${ }^{2}$ Kumamoto Rehabilitation Hospital, Kikuchi-Gun, Japan; ${ }^{3}$ Department of Neurology, Juntendo University School of Medicine, Tokyo, Japan; ${ }^{4}$ Pfizer Japan Inc., Tokyo, Japan
Correspondence: Kenji Onouchi Aida Memorial Rehabilitation Hospital, 360 Dochi, Moriya, Ibaraki 302-0I I2, Japan Tel +8I 297486 III

Fax +8I 297486116

Email kenji-o@ya2.so-net.ne.jp
Purpose: Studies of pregabalin for the treatment of central neuropathic pain have been limited to double-blind trials of 4-17 weeks in duration. The purpose of this study was to assess the long-term safety and tolerability of pregabalin in Japanese patients with central neuropathic pain. The efficacy of pregabalin was also assessed as a secondary measure.

Patients and methods: This was a 53-week, multicenter, open-label trial of pregabalin (150-600 mg/day) in Japanese patients with central neuropathic pain due to spinal cord injury, multiple sclerosis, or cerebral stroke.

Results: A total of 103 patients received pregabalin (post-stroke $=60$; spinal cord injury $=38$; and multiple sclerosis $=5)$. A majority of patients $(87.4 \%)$ experienced one or more treatment-related adverse events, most commonly somnolence, weight gain, dizziness, or peripheral edema. The adverse event profile was similar to that seen in other indications of pregabalin. Most treatmentrelated adverse events were mild (89.1\%) or moderate $(9.2 \%)$ in intensity. Pregabalin treatment improved total score, sensory pain, affective pain, visual analog scale (VAS), and present pain intensity scores on the Short-Form McGill Pain Questionnaire (SF-MPQ) and ten-item modified Brief Pain Inventory (mBPI-10) total score at endpoint compared with baseline. Improvements in SF-MPQ VAS and mBPI-10 total scores were evident in all patient subpopulations. Mean changes from baseline in SF-MPQ VAS and mBPI-10 scores at endpoint were -20.1 and -1.4, respectively.

Conclusion: These findings demonstrate that pregabalin is generally well tolerated and provides sustained efficacy over a 53-week treatment period in patients with chronic central neuropathic pain.

Keywords: clinical trial, spinal cord injury, multiple sclerosis, cerebral stroke

\section{Introduction}

Neuropathic pain results from damage to the nervous system and may be classified as central or peripheral, depending on the site of the lesion. ${ }^{1}$ Central neuropathic pain is estimated to occur in approximately $40 \%$ of patients following spinal cord injury ${ }^{2} 27 \%$ of patients with multiple sclerosis; ${ }^{3}$ and $8 \%$ of patients following stroke. ${ }^{4}$ Such pain is often severe, chronic, and refractory to pharmacologic treatment, which may include anticonvulsants, antidepressants, analgesics, and antispasticity medications. ${ }^{1,5-7}$ As a result, central neuropathic pain has a substantial negative impact on patient function and overall quality of life..$^{8,9}$

Pregabalin, an $\alpha 2 \delta$ ligand, is approved for the treatment of peripheral neuropathic pain in the European Union and Japan, and for peripheral neuropathic pain due to diabetic peripheral neuropathy (DPN) or postherpetic neuralgia (PHN) in the United States. 
Pregabalin is also approved for the treatment of central neuropathic pain in the European Union and, more recently, for the treatment of neuropathic pain associated with spinal cord injury in the United States. Studies of pregabalin for the treatment of central neuropathic pain, however, have been limited to double-blind studies of 4-17 weeks in duration. ${ }^{10-13}$ The purpose of the current 53-week, open-label study was to assess the long-term safety and tolerability of pregabalin in Japanese patients with central neuropathic pain due to spinal cord injury, multiple sclerosis, or cerebral stroke. The long-term efficacy of pregabalin in this population was also examined.

\section{Patients and methods Inclusion and exclusion criteria}

Patients with central neuropathic pain due to spinal cord injury were recruited from a previous short-term trial of pregabalin (ClinicalTrials.gov identifier: NCT00407745). To be eligible for participation in the current 53-week study (ClinicalTrials.gov identifier: NCT01202227), patients must have completed all efficacy assessments upon conclusion of the previous short-term study. The previous study was conducted in ten countries, but only Japanese patients were recruited for the current study. The results of this previous study, as well as a full list of inclusion/exclusion criteria, are published elsewhere. ${ }^{12}$ Patients aged $\geq 18$ years, with central neuropathic pain of $\geq 6$ months in duration due to cerebral stroke or multiple sclerosis were also eligible for the current study, provided they had a score of $\geq 40 \mathrm{~mm}$ on the $100 \mathrm{~mm}$ visual analog scale ([VAS] from $0=$ no pain to $100=$ worst possible pain) of the Short-Form McGill Pain Questionnaire (SF-MPQ) at screening and at the start of the treatment phase. Post-stroke patients were also required to have involvement of the cutaneous sensory pathway in the stroke location, confirmed by computerized tomography or magnetic resonance imaging, and neuropathic pain developed at the site of the post-stroke sensory disturbance. Patients with multiple sclerosis had to be diagnosed according to the McDonald Diagnostic Criteria ${ }^{14}$ and were required to score $<6.5$ on the Expanded Disability Status Scale. ${ }^{15}$

Key exclusion criteria included the following: having experienced a serious treatment-related adverse event (AE) during the previous randomized controlled trial (patients with spinal cord injury only); atrial fibrillation during 12-lead electrocardiogram (ECG) at the first visit (post-stroke patients only); uncontrolled diabetes, hypertension, or hyperlipidemia (post-stroke patients only); dementia, serious aphasia, or hemispatial neglect (post-stroke patients only); a diagnosis of neuromyelitis optica (patients with multiple sclerosis only); serious nociceptive pain, musculoskeletal pain, or painful tonic convulsion (patients with multiple sclerosis only); peripheral neuropathic pain or trigeminal neuralgia (patients with multiple sclerosis only); other severe pain that could confound the assessment of central neuropathic pain; participation in a previous trial of pregabalin (except patients with spinal cord injury); intolerance or hypersensitivity to pregabalin or gabapentin; and a documented retinal abnormality or use of a retinotoxic agent.

Patients were required to discontinue gabapentin or cannabinoids at least 7 days before screening, and pregabalin at least 60 days prior. Nonsteroidal anti-inflammatory drugs, cyclooxygenase- 2 inhibitors, and acetaminophen $(\leq 1.5 \mathrm{~g} /$ day in Japan) were permitted as rescue therapy. Antidepressants were permitted if the patient was on a stable dose within 30 days prior to first visit.

\section{Study design}

This 53-week, open-label study was conducted from 2010 to 2012 at 26 medical centers in Japan. The study protocol was approved by the appropriate institutional review board or independent ethics committee at each participating investigational center, and all patients provided written informed consent prior to entering the study. This study was conducted in compliance with the ethical principles originating in or derived from the Declaration of Helsinki and in compliance with all International Conference on Harmonization Good Clinical Practice Guidelines.

The study was composed of three phases: a 2-week screening period for newly enrolled patients; a 52-week open-label treatment period; and a 1-week taper period. All patients received $75 \mathrm{mg}$ of pregabalin on day 1 of the 52 -week treatment period, and $150 \mathrm{mg}$ /day for the remainder of the first week. Based on patient tolerability, the dose of pregabalin could be increased to $300 \mathrm{mg} /$ day on day 8 , $450 \mathrm{mg}$ /day on day 15, and $600 \mathrm{mg}$ /day on day 22. Weekly dose adjustments were allowed from day 8 until day 29. Ideally, after day 29, patients received their optimized dose of pregabalin until the end of the 52-week treatment period. However, in consideration of efficacy and safety, a singlelevel ( $\pm 150 \mathrm{mg} /$ day) dose adjustment was allowed at each visit during the dose maintenance period (weeks $4,8,12$, $20,28,36$, and 44). After the week 52 visit, patients were tapered off pregabalin over a 1 -week period.

\section{Safety and tolerability measures}

The primary endpoint of this study was to assess the safety and tolerability of pregabalin in patients with central 
neuropathic pain. The safety profile of pregabalin was assessed based on observed and reported AEs, which were evaluated throughout the study by the investigator for severity and relationship to treatment. Additional safety measures included ophthalmologic, neurologic, and physical examinations with assessment of vital signs; edema and deep vein thrombosis assessments; clinical laboratory testing; and 12-lead ECG.

\section{Efficacy measures}

A secondary endpoint of this study was to examine the efficacy of pregabalin patients with central neuropathic pain. Efficacy was assessed using the SF-MPQ ${ }^{16}$ and the ten-item modified Brief Pain Inventory (mBPI-10). ${ }^{17,18}$ The SF-MPQ is a patient-reported instrument that consists of 15 pain descriptors (eleven sensory and four affective), which are rated on a scale from 0-3. Composite SF-MPQ total score ranges from $0-45$, while the sensory and affective scores range from 0-33 and 0-12, respectively. The SF-MPQ also provides a present pain index (PPI) score that ranges from $0-5$, and a VAS for pain that ranges from $0-100 \mathrm{~mm}$. For all SF-MPQ items, higher scores indicate greater pain severity. The SFMPQ was completed at each study visit. The mBPI-10 is a patient-reported instrument that assesses pain interference with functional activities over the previous week. Items are measured on a scale from $0-10$, with higher scores indicating greater interference. Total score was calculated by averaging the ten individual items listed on the mBPI-10. The mBPI-10 was completed at week 1 and week 52, or at the time of study discontinuation.

\section{Statistical analysis}

All subjects who received at least one dose of study medication were included in the safety analysis. The efficacy analyses included all subjects who received at least one dose of study medication and had both baseline and at least one post-baseline efficacy measurement. All safety and efficacy measures were summarized descriptively and no inferential testing was performed. A last-observation-carried-forward approach for missing values was used to summarize SF-MPQ scores at endpoint.

\section{Results \\ Patients and treatment}

A total of 112 patients were screened and 103 patients received pregabalin treatment (Figure 1). Of the patients receiving pregabalin, 60 had post-stroke pain, 38 had spinal cord injury-related pain, and five had pain related to multiple sclerosis (Table 1). Median (range) treatment duration for

\begin{tabular}{|c|c|c|c|c|}
\hline & \multicolumn{4}{|c|}{ Assessed for eligibility $(n=112)$} \\
\hline & $\rightarrow$ & \multicolumn{3}{|c|}{$\begin{array}{l}\text { Did not meet inclusion criteria }(n=6) \\
\text { Declined to participate }(n=1) \\
\text { Other }(n=1)^{\star}\end{array}$} \\
\hline & \multicolumn{4}{|c|}{ Assigned to treatment $(n=104)$} \\
\hline & 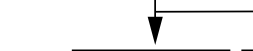 & 7 & 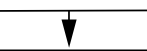 & 7 \\
\hline & $\begin{array}{c}\text { All } \\
\text { patients }\end{array}$ & $\begin{array}{l}\text { Post } \\
\text { stroke }\end{array}$ & $\begin{array}{l}\text { Spinal cord } \\
\text { injury }\end{array}$ & $\begin{array}{l}\text { Multiple } \\
\text { sclerosis }\end{array}$ \\
\hline & $\downarrow$ & 1 & 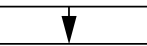 & $\downarrow$ \\
\hline Assigned to treatment & 104 & 60 & 39 & 5 \\
\hline Received treatment & 103 & 60 & 38 & 5 \\
\hline Did not receive treatment & 1 & 0 & 1 & 0 \\
\hline \multirow[t]{2}{*}{ Did not meet inclusion criteria } & 1 & 0 & 1 & 0 \\
\hline & 7 & $\nabla$ & 7 & 7 \\
\hline Completed treatment & $84(81.6 \%)$ & $47(78.3 \%)$ & $32(84.2 \%)$ & $5(100 \%)$ \\
\hline Discontinued treatment & $19(18.4 \%)$ & $13(21.7 \%)$ & $6(15.8 \%)$ & 0 \\
\hline Adverse event & 16 & 12 & 4 & 0 \\
\hline Subject withdrew & 2 & 0 & 2 & 0 \\
\hline \multirow[t]{2}{*}{ Insufficient response } & 1 & 1 & 0 & 0 \\
\hline & $\downarrow$ & $\downarrow$ & 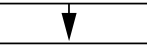 & $\gamma$ \\
\hline \multicolumn{5}{|l|}{ Analyzed } \\
\hline Safety & 103 (100\%) & $60(100 \%)$ & $38(100 \%)$ & $5(100 \%)$ \\
\hline Efficacy & $103 \quad(100 \%)$ & $60(100 \%)$ & $38(100 \%)$ & $5(100 \%)$ \\
\hline
\end{tabular}

Figure I Patient disposition.

Note: *Patient died of pneumonia prior to being assigned to study treatment. 
Table I Patient demographics and clinical characteristics ${ }^{\mathrm{a}}$

\begin{tabular}{|c|c|c|c|c|}
\hline Characteristic & $\begin{array}{l}\text { All patients } \\
(n=103)\end{array}$ & $\begin{array}{l}\text { Post-stroke } \\
(n=60)\end{array}$ & $\begin{array}{l}\text { Spinal cord injury } \\
(n=38)\end{array}$ & $\begin{array}{l}\text { Multiple sclerosis } \\
(n=5)\end{array}$ \\
\hline \multicolumn{5}{|l|}{ Sex, $n$} \\
\hline Male & 81 & 42 & 35 & 4 \\
\hline Female & 22 & 18 & 3 & I \\
\hline \multicolumn{5}{|l|}{ Age, years } \\
\hline Mean (SD) & $57.9(11.2)$ & $61.7(8.5)$ & $53.6(12.7)$ & $44.6(5.7)$ \\
\hline Range & $29-80$ & $44-80$ & $29-75$ & $39-54$ \\
\hline$\geq 65$ years, $n$ & 28 & 22 & 6 & 0 \\
\hline \multicolumn{5}{|l|}{ Weight, kg } \\
\hline Mean (SD) & $64.5(10.7)$ & $63.6(10.1)$ & $66.4(11.9)$ & $59.8(6.7)$ \\
\hline \multicolumn{5}{|l|}{$\mathrm{BMI}, \mathrm{kg} / \mathrm{m}^{2}$} \\
\hline Mean (SD) & $23.9(3.3)$ & $24.4(3.4)$ & $23.3(3.4)$ & $22.8(\mathrm{I} .3)$ \\
\hline \multicolumn{5}{|c|}{ Duration since diagnosis, months } \\
\hline Mean & - & 63.7 & 127.3 & 70.2 \\
\hline Median & - & 38.7 & 83.1 & 64.4 \\
\hline Range & - & $6.2-274.0$ & $12.4-403.2$ & $6.8-173.7$ \\
\hline \multicolumn{5}{|c|}{ Duration of pain, ${ }^{\mathrm{b}}$ months } \\
\hline Mean & 80.1 & 59.0 & 116.9 & 53.4 \\
\hline Median & 45.0 & 35.0 & 71.0 & 64.0 \\
\hline Range & $6-396$ & $6-274$ & $10-396$ & 6-94 \\
\hline
\end{tabular}

Notes: aAll patients reported "Asian" as race; 'central neuropathic pain.

Abbreviations: BMI, body mass index; SD, standard deviation.

all patients combined was 367 (3-386) days. In total, $61.2 \%$ of patients received $\geq 365$ days of study drug and $89.3 \%$ received $\geq 181$ days of study drug. The mean (standard deviation $[\mathrm{SD}]$ )/median (range) daily dose of pregabalin at final visit was $383(158) / 450(150-600) \mathrm{mg} /$ day. Overall, $5.8 \%$ of patients received a maximum daily dose of pregabalin of $150-299 \mathrm{mg} /$ day; $23.3 \%$ received $300-449 \mathrm{mg} /$ day; $28.2 \%$ received $450-599 \mathrm{mg}$ /day; and $42.7 \%$ received $600 \mathrm{mg} /$ day. Common concomitant drug treatments are shown in Table 2. All patients received at least one concomitant treatment of any kind, while $81.6 \%$ received at least one concomitant drug treatment related to pain management. The most common concomitant pain medications were loxoprofen sodium and ketoprofen, which were received by $30 \%$ and $29 \%$ of patients, respectively.

\section{Safety and tolerability}

A majority of patients (87.4\%) experienced at least one treatment-related AE, most commonly somnolence, weight gain, dizziness, or peripheral edema (Tables 3 and 4). However, most treatment-related AEs were mild-to-moderate in intensity and few patients discontinued due to an AE. Of the 229 treatment-related AEs reported, 204 (89.1\%) were characterized as mild, $21(9.2 \%)$ were characterized as moderate, and $4(1.7 \%)$ were characterized as severe. Severe treatment-related AEs included one instance each of feeling abnormal (post-stroke patient), ataxia (post-stroke patient), cerebral hemorrhage (post-stroke patient), and somnolence (patient with spinal cord injury). The instance of cerebral hemorrhage was characterized as the only treatment-related serious AE reported in the study. No deaths were reported during the study.

Table 2 Concomitant medications used by patients on pregabalin $(\mathrm{N}=103)$ during the study ${ }^{\mathrm{a}}$

\begin{tabular}{|c|c|}
\hline Concomitant medication & n (\%) \\
\hline Any pain medication & $84(81.6)$ \\
\hline Loxoprofen sodium & $31(30.1)$ \\
\hline Ketoprofen & $30(29.1)$ \\
\hline Mecobalamin & $15(14.6)$ \\
\hline Clonazepam & $14(13.6)$ \\
\hline Carbamazepine & $\mathrm{II}(\mathrm{I} 0.7)$ \\
\hline Any non-pain medication & $103(100.0)$ \\
\hline Magnesium oxide & $31(30.1)$ \\
\hline Amlodipine besilate & $30(29.1)$ \\
\hline Rebamipide & $22(21.4)$ \\
\hline Sennoside A + B & $21(20.4)$ \\
\hline Cefcapene pivoxil hydrochloride & $19(18.4)$ \\
\hline Pl gran & $17(16.5)$ \\
\hline Influenza vaccine & $16(15.5)$ \\
\hline Acetylsalicylic acid & $13(12.6)$ \\
\hline Etizolam & $12(11.7)$ \\
\hline Rosuvastatin calcium & $12(11.7)$ \\
\hline Teprenone & $12(11.7)$ \\
\hline Candesartan cilexetil & II (I0.7) \\
\hline Famotidine & II (10.7) \\
\hline Loxoprofen sodium & II (I0.7) \\
\hline New lecicarbon & II (I0.7) \\
\hline Valisone-G & II (I0.7) \\
\hline Valsartan & II (I0.7) \\
\hline
\end{tabular}

Note: a Used by more than $10 \%$ of patients. 
Table 3 Number of treatment-emergent adverse events (AEs)

\begin{tabular}{lll}
\hline & $\begin{array}{l}\text { Treatment-related, } \\
\mathbf{n}(\%)\end{array}$ & $\begin{array}{l}\text { All causalities, } \\
\mathbf{n}(\%)\end{array}$ \\
\hline Patients with $\geq$ I AE & $90(87.4)$ & $100(97.1)$ \\
Patients with $\geq$ I serious AE & $\mathrm{I}(1.0)$ & $19(18.4)$ \\
Patients with $\geq$ I severe AE & $4(3.9)$ & $12(11.7)$ \\
Discontinuations due to AE & $13(12.6)$ & $16(15.5)$ \\
Deaths & 0 & 0 \\
\hline
\end{tabular}

The mean (SD) change in body weight from baseline was $1.7(3.1) \mathrm{kg}$ in all patients combined. Fourteen patients (13.6\%) experienced clinically important weight gain at last observation, which was defined as an increase of $\geq 7 \%$ from baseline. In addition, 25 patients (24.3\%) experienced clinically important weight gain at any point during the study, compared with only two patients $(1.9 \%)$ who experienced clinically important weight loss (a decrease of $\geq 7 \%$ ).

Clinically significant increase in systolic and diastolic blood pressures (both measured sitting) occurred in one patient each at endpoint. There was no change in pulse rate that was clinically significant. There were two patients with clinically significant abnormal ECG findings at endpoint. Atrial fibrillation was reported as a treatment-related $\mathrm{AE}$ at endpoint for one of these patients; however, the fibrillation was mild in terms of severity and resolved after cessation of treatment.

Major laboratory abnormalities (those with an incidence $\geq 10 \%$, regardless of whether baseline levels were within the reference range) were increased triglycerides, increased low-density lipoprotein cholesterol, and urinary occult blood positive. There was no laboratory abnormality reported as a serious AE. There were no other clinically significant findings related to laboratory tests, vital signs, ECGs, or physical examinations during the study.

\section{Efficacy}

In all patients combined, pregabalin treatment was associated with improvements from baseline on all predefined efficacy measures at endpoint. Mean improvement in SF-MPQ total score was -4.6 at endpoint (Table 5). Mean (SD) improvements in SF-MPQ sensory and affective scores were -3.6 (6.2) and -1.0 (2.7), respectively (Table 5). SF-MPQ VAS pain score was improved by $-20.1(25.2) \mathrm{mm}$ and the SF-MPQ PPI was improved by -0.7 (1.1) (Table 5). Improvements in SF-MPQ VAS score were evident at week 2 and were sustained throughout the treatment period (Figure 2). Finally, a-1.4 (2.4) improvement was observed in mBPI-10 total score at endpoint (Table 6).

Improvements in SF-MPQ total score, SF-MPQ sensory pain score, SF-MPQ affective pain score, and SF-MPQ PPI score were evident in patients with post-stroke pain or spinal cord injury-related pain, but not in patients with multiple sclerosis (Table 5). Improvements in SF-MPQ VAS score and $\mathrm{mBPI}-10$ total score were evident in all patient populations, including those with pain due to multiple sclerosis (Tables 5 and 6).

\section{Discussion}

The results of this open-label study support the safety and efficacy of pregabalin for the management of central

Table 4 Summary of common treatment-emergent adverse events (AEs)

\begin{tabular}{|c|c|c|c|c|c|c|}
\hline \multirow[t]{2}{*}{ Common AEs ${ }^{a}$} & \multicolumn{3}{|c|}{ Treatment-related } & \multicolumn{3}{|c|}{ All causalities } \\
\hline & n (\%) & $\begin{array}{l}\text { Time to } \\
\text { onset, days }\end{array}$ & $\begin{array}{l}\text { Duration, } \\
\text { days }\end{array}$ & n (\%) & $\begin{array}{l}\text { Time to } \\
\text { onset, days }\end{array}$ & $\begin{array}{l}\text { Duration, } \\
\text { days }\end{array}$ \\
\hline Somnolence & $50(48.5)$ & 20.0 & 166.5 & $53(5 \mathrm{I} .5)$ & 18.0 & 150.0 \\
\hline Weight gain & $29(28.2)$ & 64.0 & 274.0 & $31(30.1)$ & 82.0 & 233.0 \\
\hline Dizziness & $23(22.3)$ & 5.0 & 70.0 & $24(23.3)$ & 5.0 & 63.5 \\
\hline Peripheral edema & $18(17.5)$ & 53.5 & 164.0 & $18(17.5)$ & 39.5 & 164.0 \\
\hline Feeling abnormal & $7(6.8)$ & 1.0 & 15.0 & $7(6.8)$ & 1.0 & 15.0 \\
\hline Constipation & $5(4.9)$ & 113.0 & 260.0 & $8(7.8)$ & 32.5 & 304.5 \\
\hline Thirst & $5(4.9)$ & 20.0 & 351.0 & $7(6.8)$ & 23.0 & 346.0 \\
\hline Visual acuity reduced & $5(4.9)$ & 372.0 & 1.0 & $7(6.8)$ & 372.0 & 1.0 \\
\hline Asthenia & $3(2.9)$ & 2.0 & 38.0 & $3(2.9)$ & 2.0 & 38.0 \\
\hline Fatigue & $3(2.9)$ & 18.0 & 134.0 & $4(3.9)$ & 30.0 & 169.0 \\
\hline Hyperuricemia & $3(2.9)$ & 30.0 & 260.0 & $4(3.9)$ & 30.0 & 222.0 \\
\hline Nausea & $3(2.9)$ & 1.0 & 12.0 & $3(2.9)$ & 1.0 & 12.0 \\
\hline Neutropenia & $3(2.9)$ & 258.0 & 8.0 & $3(2.9)$ & 258.0 & 8.0 \\
\hline Neutrophil count decreased & $3(2.9)$ & 82.0 & 12.0 & $3(2.9)$ & 82.0 & 12.0 \\
\hline Vision blurred & $3(2.9)$ & 24.0 & 287.0 & $3(2.9)$ & 24.0 & 287.0 \\
\hline Renal impairment & $3(2.9)$ & 225.0 & 50.0 & $3(2.9)$ & 225.0 & 50.0 \\
\hline
\end{tabular}

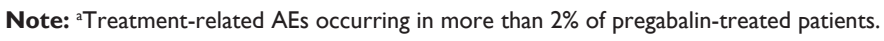


Table 5 Summary of SF-MPQ ${ }^{16}$ scores

\begin{tabular}{|c|c|c|c|c|}
\hline SF-MPQ domain & $\begin{array}{l}\text { All patients } \\
(\mathrm{N}=103)\end{array}$ & $\begin{array}{l}\text { Post-stroke } \\
(n=60)\end{array}$ & $\begin{array}{l}\text { Spinal cord } \\
\text { injury }(n=38)\end{array}$ & $\begin{array}{l}\text { Multiple sclerosis } \\
(n=5)\end{array}$ \\
\hline \multicolumn{5}{|l|}{ Total score ${ }^{a}$} \\
\hline Baseline mean (SD) & $12.2(9.1)$ & I I.8 (8.8) & I3.I (9.8) & $10.0(7.5)$ \\
\hline Endpoint mean (SD) & $7.6(8.8)$ & $6.1(6.7)$ & $9.2(10.7)$ & $13.6(11.6)$ \\
\hline \multicolumn{5}{|l|}{ Change from baseline } \\
\hline Mean (SD) & $-4.6(8.3)$ & $-5.7(8.6)$ & $-3.9(7.5)$ & $3.6(7.5)$ \\
\hline $95 \% \mathrm{Cl}$ & $(-6.2,-3.0)$ & $(-7.9,-3.5)$ & $(-6.4,-1.4)$ & $(-5.7,12.9)$ \\
\hline \multicolumn{5}{|l|}{ Sensory pain score ${ }^{b}$} \\
\hline Baseline mean (SD) & $9.5(7.1)$ & $9.0(7.0)$ & $10.4(7.5)$ & $8.8(6.3)$ \\
\hline Endpoint mean (SD) & $5.9(6.3)$ & $4.6(4.7)$ & $7.4(7.7)$ & $10.4(8.4)$ \\
\hline \multicolumn{5}{|l|}{ Change from baseline } \\
\hline Mean (SD) & $-3.6(6.2)$ & $-4.4(6.5)$ & $-3.0(5.7)$ & $1.6(4.8)$ \\
\hline $95 \% \mathrm{Cl}$ & $(-4.8,-2.4)$ & $(-6.1,-2.7)$ & $(-4.9,-1.1)$ & $(-4.3,7.5)$ \\
\hline \multicolumn{5}{|l|}{ Affective pain score ${ }^{c}$} \\
\hline Baseline mean (SD) & $2.7(2.6)$ & $2.7(2.6)$ & $2.7(2.6)$ & $1.2(1.3)$ \\
\hline Endpoint mean (SD) & I.7 (2.7) & $1.4(2.3)$ & $1.8(3.1)$ & $3.2(3.3)$ \\
\hline \multicolumn{5}{|l|}{ Change from baseline } \\
\hline Mean (SD) & $-1.0(2.7)$ & $-1.3(2.8)$ & $-0.9(2.5)$ & $2.0(2.8)$ \\
\hline $95 \% \mathrm{Cl}$ & $(-1.5,-0.5)$ & $(-2.0,-0.6)$ & $(-1.7,-0.1)$ & $(-1.5,5.5)$ \\
\hline \multicolumn{5}{|l|}{ VAS pain score } \\
\hline Baseline mean (SD) & $67.1(16.6)$ & $68.0(16.7)$ & $66.1(16.8)$ & $62.8(16.2)$ \\
\hline Endpoint mean (SD) & $47.0(26.9)$ & $41.7(27.6)$ & $53.9(24.1)$ & $58.2(28.7)$ \\
\hline \multicolumn{5}{|l|}{ Change from baseline } \\
\hline Mean (SD) & $-20.1(25.2)$ & $-26.3(26 . I)$ & $-12.2(20.5)$ & $-4.6(29.1)$ \\
\hline $95 \% \mathrm{Cl}$ & $(-25.0,-15.1)$ & $(-33.1,-19.6)$ & $(-18.9,-5.5)$ & $(-40.8,31.6)$ \\
\hline \multicolumn{5}{|l|}{ PPI score } \\
\hline Baseline mean (SD) & $2.5(1.0)$ & $2.4(1.0)$ & $2.8(\mathrm{I} .0)$ & $2.2(0.4)$ \\
\hline Endpoint mean (SD) & $1.8(1.1)$ & $1.7(1.1)$ & $2.0(1.2)$ & $2.2(0.8)$ \\
\hline \multicolumn{5}{|l|}{ Change from baseline } \\
\hline Mean (SD) & $-0.7(1.1)$ & $-0.8(1.0)$ & $-0.8(1.2)$ & $0.0(0.7)$ \\
\hline $95 \% \mathrm{Cl}$ & $(-1.0,-0.5)$ & $(-1.0,-0.5)$ & $(-1.2,-0.4)$ & $(-0.9,0.9)$ \\
\hline
\end{tabular}

Notes: aScores range from 0-45 with higher scores indicating more severe pain; 'scores range from 0-33 with higher scores indicating more severe pain; 'scores range from 0-12 with higher scores indicating more severe pain; 'scores range from 0-100 with higher scores indicating more severe pain; ${ }^{e}$ scores range from 0-5 with higher scores indicating more severe pain.

Abbreviations: Cl, confidence interval; PPI, present pain intensity; SD, standard deviation; SF-MPQ, Short-Form McGill Pain Questionnaire; VAS, visual analog scale.

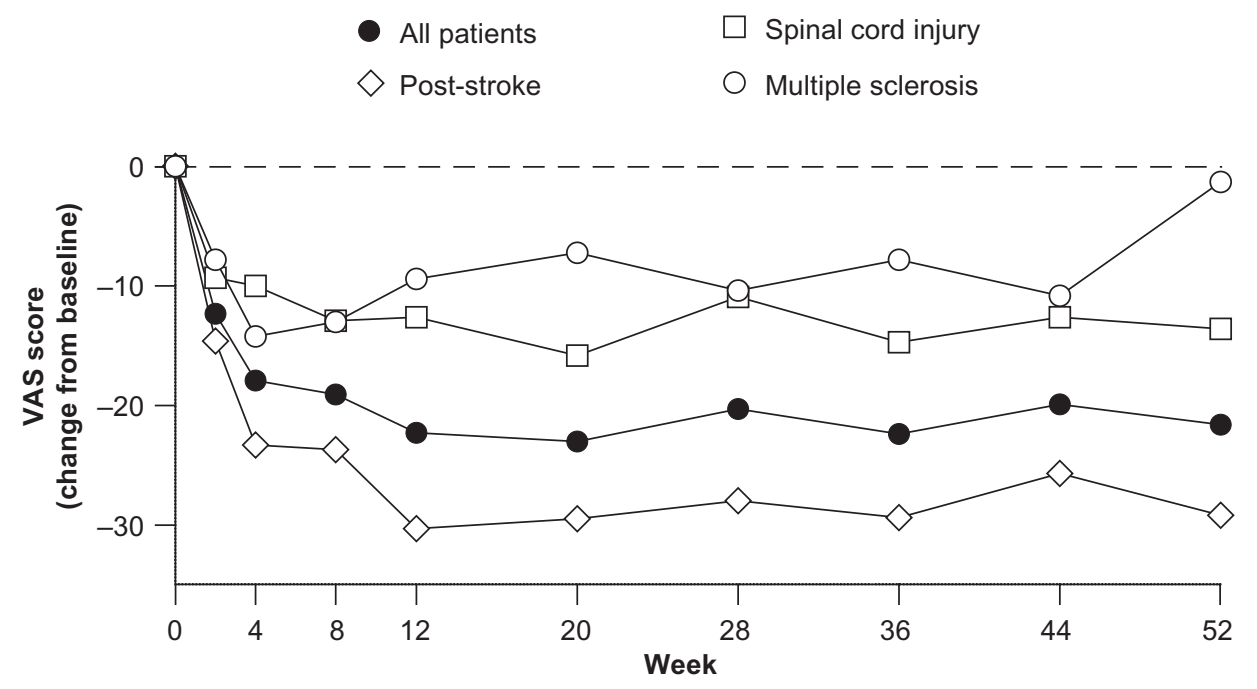

Figure 2 Short-Form McGill Pain Questionnaire ${ }^{16}$ visual analog scale (VAS) scores by week. 
Table 6 Summary of mBPI-1017,18 total scores ${ }^{\mathrm{a}}$

\begin{tabular}{|c|c|c|c|c|}
\hline mBPI-I0 score & All patients & Post-stroke & Spinal cord injury & Multiple sclerosis \\
\hline \multicolumn{5}{|l|}{ Baseline } \\
\hline $\mathrm{N}$ & 103 & 60 & 38 & 5 \\
\hline Mean (SD) & $3.9(2.4)$ & $3.8(2.5)$ & $3.9(2.1)$ & $5.0(2.1)$ \\
\hline \multicolumn{5}{|l|}{ Endpoint } \\
\hline $\mathrm{N}$ & 101 & 59 & 37 & 5 \\
\hline Mean (SD) & $2.5(2.3)$ & $2.3(2.2)$ & $2.6(2.2)$ & $3.1(3.4)$ \\
\hline \multicolumn{5}{|c|}{ Change from baseline } \\
\hline$N$ & 101 & 59 & 37 & 5 \\
\hline Mean (SD) & $-1.4(2.4)$ & $-1.5(2.6)$ & $-1.3(2.0)$ & $-1.9(3.9)$ \\
\hline $95 \% \mathrm{Cl}$ & $(-1.9,-0.9)$ & $(-2.1,-0.8)$ & $(-2.0,-0.6)$ & $(-6.8,2.9)$ \\
\hline
\end{tabular}

Note: aScores range from 0-10 with higher scores indicating greater impairment.

Abbreviations: $\mathrm{Cl}$, confidence interval; $\mathrm{mBPI}-10$, ten-item modified Brief Pain Inventory; SD, standard deviation.

neuropathic pain over a 1-year treatment period. Treatmentrelated AEs in this study were consistent with the known safety profile of pregabalin. Somnolence, however, occurred more frequently in the current population (48.5\%) than in previous studies of pregabalin for the treatment of central neuropathic pain. In previous short-term trials (13-17 weeks), somnolence was reported by $35.7 \%$ of patients with spinal cord injury ${ }^{10,12}$ and $20.9 \%$ of patients with post-stroke pain. ${ }^{11}$ The incidence of somnolence in the current study was also higher than that observed in long-term studies of pregabalin in Japanese patients with peripheral neuropathic pain due to DPN $(22.8 \%)^{19}$ or PHN (15.1\%). ${ }^{20}$ This may be explained by the fact that benzodiazepines were allowed as concomitant treatment in the current study, whereas they were prohibited during the trials of PHN and DPN. Indeed, benzodiazepines may add to the central nervous system side effects of pregabalin and have been shown to increase the rate of somnolence when used in addition to pregabalin. ${ }^{21}$ Most instances of treatment-related somnolence $(\mathrm{n}=50)$ in the current study were mild in severity (mild $n=43$, moderate $n=6$, severe $\mathrm{n}=1$ ), and only three patients discontinued treatment due to somnolence.

The frequency of weight gain as a treatment-related $\mathrm{AE}$ in the current study (28.2\%) was higher than that reported for previous short-term studies of pregabalin in patients with spinal cord injury $(3.3 \%)^{10,12}$ or post-stroke pain $(5.5 \%) .{ }^{11}$ The current observed incidence of weight gain as an $\mathrm{AE}$ was also higher than that reported during long-term, open-label trials of pregabalin in Japanese patients with DPN $(22.0 \%)^{19}$ or PHN (13.5\%). ${ }^{20}$ Likewise, the frequency of treatment-related peripheral edema was higher in the current trial (17.5\%) than in the previous short-term trials in patients with spinal cord injury $(8.8 \%)^{10,12}$ or post-stroke pain $(8.2 \%) .{ }^{11}$ However, the incidence of peripheral edema reported here was similar to that reported for long-term studies of pregabalin patients with DPN (15.4\%) ${ }^{19}$ or PHN (16.7\%). ${ }^{20}$ Most instances of treatment-related weight gain (mild $n=28$, moderate $n=1$, severe $n=0$ ) and peripheral edema (mild $n=17$, moderate $\mathrm{n}=1$, severe $\mathrm{n}=0$ ) in the current study were mild in severity and no patients discontinued treatment due to either weight gain or peripheral edema.

In all patients combined, pregabalin treatment improved pain at endpoint compared with baseline. This was evident for all aspects of the SF-MPQ, which assesses severity of pain, as well as the mBPI-10, which measures how pain interferes with patient function. Notably, improvements in SF-MPQ VAS score were evident as early as week 2 and were sustained throughout the full treatment period. Improvements in SF-MPQ VAS score and mBPI-10 total score were evident in each patient population. The proportion of patients achieving at least a 30\% decrease in SF-MPQ VAS pain score from baseline to endpoint was $40.0 \%$ in multiple sclerosis patients, $28.9 \%$ in spinal cord injury patients, and $50.0 \%$ in post-stroke patients. Improvements in other aspects of the SF-MPQ were observed in patients with spinal cord injury and post-stroke pain, but were not evident in patients with multiple sclerosis. However, the small number of patients with multiple sclerosis enrolled in this study ( $\mathrm{n}=5$ ) makes it difficult to draw conclusions regarding the effects of pregabalin in this specific patient population.

All patients received concomitant medication of some kind, and $81.6 \%$ received concomitant medication specifically for the treatment of pain (Table 2). The use of concomitant pain medication was similar in each patient population (post-stroke $=80 \%$, spinal cord injury $=84 \%$, and multiple sclerosis $=80 \%$ ). In an ad hoc exploratory analysis, mean 
(SD) change in SF-MPQ VAS pain score from baseline to endpoint was similar in patients receiving concomitant pain medicine ( -21.0 [24.50]) compared to those who did not receive concomitant pain medication $(-15.7$ [28.4]). These data suggest that pregabalin reduces central neuropathic pain whether it is used alone or as part of a combination therapy.

In previous trials enrolling patients with central neuropathic pain due to spinal cord injury, pregabalin treatment significantly reduced pain scores at endpoint (13-17 weeks) compared with placebo. ${ }^{10,12}$ Such improvements were seen as early as week 1 and were sustained throughout the treatment period. Another previous study enrolled patients with central neuropathic pain of different etiologies, including patients with pain due to spinal cord injury, stroke, thalamus lesion, or other brainstem pathology. ${ }^{13}$ In that particular study, pregabalin treatment significantly improved VAS pain score over placebo in the combined study population. Finally, in a previous trial enrolling patients with central post-stroke pain, pregabalin treatment decreased pain scores from baseline to endpoint, but these improvements in pain were not statistically different from placebo. ${ }^{11}$ The lack of significance, however, was likely due to a large placebo effect on pain in that study. ${ }^{11}$ Overall, our data are in agreement with previous short-term, placebo-controlled studies of pregabalin and provide further support for its efficacy in the treatment of central neuropathic pain.

As with all clinical trials, limitations related to study design need to be considered. For example, the open-label design may bias patient assessment of pain because of their inherent desire to improve. Additionally, the relatively small sample size, particularly for patients with multiple sclerosis, limits the extent to which our findings can be generalized beyond the specific populations included in our study. Finally, our interpretation of our efficacy results is constrained by the lack of a placebo control or active comparator.

\section{Conclusion}

Overall, our findings demonstrate that pregabalin is generally well tolerated over a 52-week treatment period in patients with chronic central neuropathic pain. Though descriptive in nature, our data also suggest that pregabalin is effective in the treatment of central neuropathic pain out to 1 year.

\section{Acknowledgments}

The authors would like to thank Makoto Suzuki, $\mathrm{PhD}$ and Takehiko Kaneko, MD (both former employees of Pfizer Japan Inc.) for their contributions as development lead for pregabalin in Japan (M Suzuki) and clinical lead for the study (T Kaneko), and for their contributions to early drafts of this manuscript. The authors would also like to thank all of the investigators from the Pregabalin Study Group who recruited patients for this study. Hokkaido Chuo Rosai Hospital Sekison Center: Dr Kota Suda, Dr Haruki Ueda, Dr Hiroshi Moridaira, Dr Tomomichi Kajino; Senboku Kumiai General Hospital: Dr Shinichi Goto, Dr Jutaro Umehara, Dr Shinichi Sato; National Hospital Organization Yamagata Hospital: Dr Shiho Toyooka, Dr Hiroshi Orui; Sendai Pain Clinic: Dr Hisashi Date, Dr Hidekazu Watanabe, Dr Noriko Takiguchi, Dr Tomofumi Chiba; Ibaraki Seinan Medical Center Hospital: Dr Hiroshi Akaogi, Dr Haruka Tanaka; Kitasato University Kitasato Institute Medical Center Hospital: Dr Takao Sagiuchi, Dr Shigeyuki Osawa; Jukoukai Hospital: DrYuji Inokuchi; Uchida Rehabilitation Orthopedic Clinic: Dr Ryusei Uchida; National Hospital Organization Niigata National Hospital: Dr Takashi Nakajima, Dr Izumi Aida, Dr Masamichi Miyoshi, Dr Shinya Higuchi, Dr Yosuke Yonemochi; Kimura Clinic: Dr Tomomasa Kimura; Brain Attack Center Ota Memorial Hospital: Dr Taisei Ota, Dr Katsuyasu Suwa, Dr Nobuharu Nishihara, Dr Tatsuya Ohtonari; Nakamura Hospital: Dr Masahiko Nakamura, Dr Eisuke Nakamura, Dr Kengo Marui; Hakodate Central General Hospital: Dr Keiichi Shigenobu, Dr Fumihiro Oha; Chubu Rosai Hospital: Dr Fumihiko Kato, Dr Hirotaka Tanaka, Dr Keigo Ito, Dr Masaaki Machino, Dr Yasutsugu Yukawa; National Hospital Organization Murayama Medical Center: Dr Masanobu Shioda, Dr Daisuke Matsuura, Dr Haruka Sugiyama, Dr Harukazu Saito, Dr Hideaki Murakami, Dr Hiroyuki Katoh, Dr Katsuhiro Mizuno, Dr Kazutaka Igarashi, Dr Kentaro Fukuda, Dr Masafumi Machida, Dr Masakazu Takemitsu, Dr Mikiko Shirai, Dr Narihito Nagoshi, Dr Osamu Uemura, Dr Shingo Iizuka, Dr Shinichiro Iwata, Dr Shinjiro Kaneko, Dr Takeshi Ikegami, Dr Toshiki Mori, Dr Yoshihiro Sasazaki, Dr Yukio Nakamura; Kumamoto Rehabilitation Hospital: Dr Hiroaki Koga, Dr Satoko Saito, Dr Chika Tanaka; Spinal Injuries Center: Dr Takayoshi Ueta, Dr Takeshi Maeda; Kamitsuga General Hospital: Dr Yoshikazu Tsuneizumi, Dr Kazuyo Yamauchi, Dr Osamu Ikeda, Dr Takeo Furuya, Dr Tomonori Yamauchi; Okitama Public General Hospital: Dr Masahiro Hayashi, Dr Fumiaki Goto, Dr Hiroshi Hasegawa; General Hanamaki Hospital: Dr Kimiaki Utsugisawa, Dr Yuriko Nagane; Kohnan Hospital: Dr Eisuke Furui, Dr Kenichi Tsukita, Dr Kenta Kawata, Dr Ryo Itabashi, Dr Shigeru Sato, Dr Shoichiro Sato, Dr Yukako Yazawa; Aida Kinen Rehabilitation Hospital: Dr Kenji Onouchi, Dr Aya Saito, 
Dr Etsuko Noguchi, Dr Jun Akanuma, Dr Osamu Takahashi, Dr Toshihiko Atobe, Dr Yasushi Makiyama, Dr Yoshiaki Yamamoto; Go Neurosurgical Clinic: Dr Yoshinori Go, Dr Takuo Kusano; Nagoya Kyoritsu Clinic: Dr Tsukasa Tamaru, Dr Hiroshi Hori, Dr Takanobu Toriyama, Dr Yoshiko Tamaru, Dr Yoshinori Ookawa; Kobe Tokushukai Hospital: Dr Satoshi Uramoto, Dr Tsunekazu Takagi, Dr Mami Kidera; Tokushima University Hospital: Dr Yuka Terasawa, Dr Hiromi Inoue, Dr Kenta Sato, Dr Motohiro Hirasawa, Dr Ryuji Kaji, Dr Takahiro Furukawa, Dr Waka Sakai, Dr Yoshitaka Kurashiki, Dr Yuishin Izumi; Juntendo University Hospital: Dr Kazumasa Yokoyama, Dr Yasushi Shimo, Dr Yuji Tomizawa, Dr Yumiko Motoi.

\section{Disclosure}

Editorial/medical writing support was provided by Matt Soulsby, PhD, of Engage Scientific Solutions and was funded by Pfizer Inc. This study was funded by Pfizer Inc. Tamotsu Yoshiyama is a full-time employee of Pfizer Inc. The authors report no other conflicts of interest in this work.

\section{References}

1. Vranken JH. Mechanisms and treatment of neuropathic pain. Cent Nerv Syst Agents Med Chem. 2009;9:71-78.

2. Siddall PJ, McClelland JM, Rutkowski SB, Cousins MJ. A longitudinal study of the prevalence and characteristics of pain in the first 5 years following spinal cord injury. Pain. 2003;103:249-257.

3. Osterberg A, Boivie J, Thuomas KA. Central pain in multiple sclerosis - prevalence and clinical characteristics. Eur J Pain. 2005;9: 531-542.

4. Andersen G, Vestergaard K, Ingeman-Nielsen M, Jensen TS. Incidence of central post-stroke pain. Pain. 1995;61:187-193.

5. Tesell RW, Mehta S, Aubut JA, et al; Spinal Cord Injury Rehabilitation Evidence Research Team. A systematic review of pharmacologic treatments of pain after spinal cord injury. Arch Phys Med Rehabil. 2010;91: 816-831.

6. Solaro C, Messmer Uccelli M. Pharmacological management of pain in patients with multiple sclerosis. Drugs. 2010;70:1245-1254.

7. Klit H, Finnerup NB, Jensen TS. Central post-stroke pain: clinical characteristics, pathophysiology, and management. Lancet Neurol. 2009;8:857-868.

Journal of Pain Research

\section{Publish your work in this journal}

The Journal of Pain Research is an international, peer-reviewed, open access, online journal that welcomes laboratory and clinical findings in the fields of pain research and the prevention and management of pain. Original research, reviews, symposium reports, hypothesis formation and commentaries are all considered for publication.
8. Widerström-Noga EG, Felipe-Cuervo E, Yezierski RP. Chronic pain after spinal injury: interference with sleep and daily activities. Arch Phys Med Rehabil. 2001;82:1571-1577.

9. Saverino A, Solaro C. Pain in individuals with multiple sclerosis, knee prosthesis, and post-herpetic neuralgia: learning from focus group patients' experience. Clin J Pain. 2012;28:300-308.

10. Siddall PJ, Cousins MJ, Otte A, Griesing T, Chambers R, Murphy TK. Pregabalin in central neuropathic pain associated with spinal cord injury: a placebo-controlled trial. Neurology. 2006;67: 1792-1800.

11. Kim JS, Bashford G, Murphy TK, Martin A, Dror V, Cheung R. Safety and efficacy of pregabalin in patients with central post-stroke pain. Pain. 2001;152:1018-1023.

12. Cardenas DD, Nieshoff EC, Suda K, et al. A randomized trial of pregabalin in patients with neuropathic pain due to spinal cord injury. Neurology. 2013;80:553-539.

13. Vranken JH, Dijkgraaf MG, Kruis MR, van der Vegt MH, Hollmann NW, Heesen M. Pregabalin in patients with central neuropathic pain: a randomized, double-blind, placebo-controlled trial of a flexible-dose regimen. Pain. 2008;136:150-157.

14. Polman CH, Reingold SC, Edan G, et al. Diagnostic criteria for multiple sclerosis: 2005 revisions to the "McDonald Criteria". Ann Neurol. 2005;58:840-846.

15. Kurtzke JF. Rating neurologic impairment in multiple sclerosis: an expanded disability status scale (EDSS). Neurology. 1983;33: 1444-1452.

16. Melzack R. The short-form McGill Pain Questionnaire. Pain. 1987;30:191-197.

17. Daut RL, Cleeland CS, Flanery RC. Development of the Wisconsin Brief Pain Questionnaire to assess pain in cancer and other diseases. Pain. 1983;17:197-210.

18. Raichle KA, Osborne TL, Jensen MP, Cardenas D. The reliability and validity of pain interference measures in persons with spinal cord injury. J Pain. 2006;7:179-186.

19. Satoh J, Yagihashi S, Baba M, Suzuki M, Arakawa A, Yoshiyama T. Efficacy and safety evaluation of pregabalin treatment over 52 weeks in patients with diabetic neuropathic pain extended after a double-blind placebo-controlled trial. J Diabetes Investig. 2011;2:457-463.

20. Ogawa S, Suzuki M, Arakawa A, Yoshiyama T, Suzuki M. [Long-term efficacy and safety of pregabalin in patients with postherpetic neuralgia: results of a 52-week, open-label, flexible-dose study]. Masui. 2010;59: 961-970. Japanese.

21. Parsons B, Sanin L, Yang R, Emir B, Juhn M. Efficacy and safety of pregabalin in patients with spinal cord injury: a pooled analysis. Curr Med Res Opin. 2013;29:1675-1683.

\section{Dovepress}

The manuscript management system is completely online and includes a very quick and fair peer-review system, which is all easy to use. Visit http://www.dovepress.com/testimonials.php to read real quotes from published authors. 